\title{
Dysregulation of epigenetic related genes in Diabetic Trigger finger Patients; preliminary analysis of Patient-Derived Samples
}

https://doi.org/10.1515/bmc-2020-0020

received November 3, 2020; accepted December 1, 2020.

\begin{abstract}
Background: Trigger finger (TF), a painful condition involving a finger flexor tendon, is a common problem with a prevalence of $\sim 2-3 \%$ in the general population. However, the TF prevalence is higher among diabetic patients-ranges from $6.7 \%$ to $10 \%$. We have analyzed the expression of the extracellular matrix, inflammation, and epigenetic related genes in diabetic and non-diabetes TF. We hypothesized that Diabetes condition induces alter the expression of epigenetic modification genes in diabetic patients and one of the underlying determinants for more prevalence of $\mathrm{TF}$ in diabetic patients.
\end{abstract}

Method: Tissues from the fingers of patients with symptomatic trigger fingers were collected. We had three groups: carpal tunnel syndrome (as a control), trigger finger, and diabetic trigger finger. A quantitative realtime polymerase chain reaction was performed. The gene expression of Extracellular matrix (ECM) components [COL-I, COL-II, COL-X, Aggrecan], DNA methyltransferases enzymes (DNMT1, DNMT3), growth factors (TGF-b, IGF),

\footnotetext{
*Corresponding author: Sadanand Fulzele, Division of Endocrinology, Diabetes, and Metabolism, Augusta University, Augusta, GA 30904, E-mail: sfulzele@augusta.edu Michael Cain, Umar Ghilzai, Carlos Isales, Mark Fulcher, Sadanand Fulzele, Department of Orthopedics, Augusta University, Augusta, GA Mohamed E. Awad, Department of Oral Biology, Augusta University, Augusta, GA

Ravindra Kolhe, Ashis K. Mondal, Department of Pathology, Augusta University, Augusta, GA, 30912, USA

Carlos Isales, Sadanand Fulzele, Center for Healthy Aging, Augusta University, Augusta, GA, 30912, United States

Carlos Isales, Division of Endocrinology, Diabetes and Metabolism, Augusta University, Augusta, GA 30912, USA

Sadanand Fulzele, Cellular Biology and Anatomy, Augusta

University, Augusta, GA 30912, USA

\# Both authors contributed equally to this manuscript
}

and Histone deacetylase enzymes (HDAC1, HDAC2) were evaluated in all groups.

Results: The mRNA expression of COL-I, COL-II, Aggrecan was significantly higher in the pully A1 of diabetic patients $(\mathrm{p}=0.0164, \mathrm{p}=0.0351, \mathrm{p}=0.0399$, respectively) as compared to non-diabetic TF patients. Diabetes was associated with a significant increase in the DNMT3 expression compared to non-diabetic TF patients $(p=0.0485)$. HDAC1 and HDAC2 gene expression were up-regulated in diabetic TF than non-diabetic TF.

Conclusion: The chronic state of hyperglycemia induces epigenetic modification of gene expressions in trigger fingers. This seems to have a significant impact on the development, recurrence, and progression of trigger finger in diabetic patients.

Keywords: Trigger Finger; Diabetic; Gene expression.

\section{Introduction}

Trigger finger or stenosing tenosynovitis is one of the most common finger aliment which is a result of a size disproportion of the flexor tendons and the surrounding retinacular pulley system at the first annular (A1) pulley. TF may lead to substantial long-term disability in the form of an inability to passively manipulate the finger to achieve normal motion. The pathophysiology of TF is still not completely clear. Several studies have pointed towards the pulley as the cause versus the tendon, but the consensus is that the system undergoes inflammatory and hypercellular changes to affect the normal motion. It's widely considered that it's caused by inflammation and subsequent fibrotic narrowing of the A1 pulley, which causes pain, clicking, catching, and loss of motion of the affected finger [1,2]. However, some studies revealed the tendon is the main site of pathological inflammation is the tendon (tendinosis) [3,4]. The trigger finger is in the clinical 
practice, frequently caused by stenosing tenosynovitis at the A1 pulley. The pulley system is a complex network of ligaments against the bowstringing of the flexor tendons. Most commonly, the trigger finger involves the A1 annular pulley at the level of the distal palmar crease [5].

The condition is usually idiopathic but can be caused by overuse, previous trauma, or an underlying connective tissue disorder, such as amyloidosis, Dupuytren's contracture, or hypothyroidism [1,6]. Patients with TF represent around $2 \%$ of the general population $[1,7]$. However, the TF prevalence is higher among diabetic patients-ranges from $6.7 \%$ to $10 \%$-[8], as well as it's seen more frequently in the female population [6], typically in the $5^{\text {th }}-6^{\text {th }}$ decade of life [9]. Moreover, the incidence of subsequent trigger finger after carpal tunnel release in diabetics is $8 \%$ and $10 \%$ at 6 and 12 months postoperatively, as compared to non-diabetics at $3 \%$ and $4 \%$ [10]. Almost $80 \%$ of diabetic patients are at risk of some form of musculoskeletal inflammation, degeneration, or infection [11]. Both type 1 and type $2 \mathrm{DM}$ are at high risk of tendinopathy or tendonitis. The flexor tendons of the hand are more likely to be affected in DM. Limited function and diminished mobility are reported in approximately $50 \%$ of diabetic patients' hands [12].

Corticosteroid injection is the main pillar in managing the idiopathic stenosing tenosynovitis. It showed superiority in modifying the inflammatory response and the course of the disease, when compared to and other nonoperative treatments such as nonsteroidal anti-inflammatory drugs (NSAIDs) and splinting [1315]. However, Forty-eight percent of patients reported the recurrence of symptoms after steroid injection [16]. Diabetic patients experienced less response to conservative treatment, such as injections [17]. Also, they have a higher risk of persistent triggering after corticosteroid injection [18].

It's evident that epigenetic modifications have been implicated in the pathology of diabetes and its complications $[16,19]$. The molecular basis of epigenetic modifications is complex and includes modifications of histones, methylation of DNA, and gene regulation by non-coding RNAs [20]. Several genes have been detected to be up- or down-regulated in trigger finger, as well as other tendinosis, such as Achilles tendinitis [21]. These genes include aggrecan, biglycan, versican, decorin, collagens type 1a1 and 3a1, and matrix metalloproteinases. Epigenetic modifications are potentially reversible, and, therefore, a thorough understanding of these changes may identify new therapeutic targets for the disease. Our study is designed to examine the expression of Cytokines (TGF-b, IGF), and Extracellular matrix (ECM) components (collagen/proteoglycan aggrecan) and epigenetic related genes such as DNA methyltransferases (DNMT1, DNMT3), histone deacetylase (HDAC1, HDAC2) in diabetics and non-diabetics patient-derived TF samples. Our hypothesis relies on Diabetes-induced epigenetic modification as an underlying determinant. We have evaluated the gene expression of epigenetic regulation associated with inflammation and structural integrity.

\section{Material and Methods}

\section{Patients samples}

Specimens from fingers of patients with symptomatic trigger fingers were collected in the Department of orthopedics, Augusta University medical center, after undergoing surgery of the A1 pulley to release trigger finger. The patients who had tenderness at the A1 pulley have been categorized into two groups; Diabetic TF and non-diabetic TF. The specimens of the control group were obtained from patients with carpal tunnel syndrome (CT) because of the limitations to get specimens from healthy subjects. Specimens were transported to the laboratory, and dissected specimens were snap-frozen and kept at $80^{\circ} \mathrm{C}$.

Ethical approval: The research related to human use has been complied with all the relevant national regulations, institutional policies and in accordance the tenets of the Helsinki Declaration, and has been approved by the authors' institutional review board or equivalent committee.

Informed consent: Informed consent has been obtained from all individuals included in this study.

\section{Isolation of RNA, synthesis of CDNA, and real-time PCR:}

Total RNA was isolated from the frozen tissues using trizol method. The tissues were ground in liquid $\mathrm{N}_{2}$ with a mortar and pestle, dissolved in Trizol for RNA isolation, per manufacturer's instructions, and the quality of the RNA preparations was monitored by absorbance at 260 and $280 \mathrm{~nm}$ (Helios-Gamma, Thermo Spectronic, Rochester, NY). The RNA was then reverse-transcribed into complementary deoxyribonucleic acid (cDNA) using iScript reagents from Bio-Rad on a programmable thermal cycler (PCR-Sprint, Thermo Electron, Milford, MA). 50 ng of cDNA was amplified in each real-time 
PCR reaction using a Bio-Rad iCycler, ABgene reagents (Fisher scientific), and gene-specific primers (Table 1). Average of Glyceraldehyde-3-phosphate dehydrogenase (GAPDH) and $18 \mathrm{~S}$ was used as the internal control for normalization. Standard curves were applied for each RNA assay to produce accurate quantification of the threshold cycle $(\Delta \mathrm{Ct})$. This allows an approximate comparison of the expression levels of different targets.

\section{Statistical analysis:}

The results were shown as a mean \pm standard deviation. GraphPad Prism 5 (La Jolla, CA) was utilized to perform ANOVA with Bonferroni pair-wise comparison or unpaired t-tests as appropriate. A p-value of $<0.05$ was considered significant.

\section{Results}

\section{Extra-cellular matrix (ECM) components (collagen/proteoglycan aggrecan). To examine the associated pathological changes in ECM of trigger finger, the expression of mRNA for COL-I, COL-II, COL-X, and Aggrecan were analyzed and compared between the included groups.}

\subsection{A Carpel tunnel syndrome (CT) versus Trigger Finger (TF)}

COL-1 mRNA expression in patients with TF was significantly higher $(p$-value $=0.002)$ expression than in carpel-tunnel syndrome. Also, COL-II mRNA expression was significantly higher ( $p$-value $=0.0214)$ in TF samples, when compared to samples from CT syndrome. However, there was no significant difference between both groups in COL-X mRNA expression. Aggrecan showed the trend of up-regulation in TF patients than CT syndrome patients [Figure.1]

\section{Diabetic TF versus Non-Diabetic TF.}

The mRNA expression of COL-I ( $p$-value $=0.0164)$ and COL-II were significantly higher in the pully A1 of diabetic patients than non-diabetic TF patients. However, both diabetic and non-diabetic TF patients have approximately the same level of COL-X expression with no statistical significance. Aggrecan expression is significantly ( $p$-value $=0.039)$ up-regulated in diabetic TF, as compared to non-diabetic TF [Figure.3].

\section{DNA methyltransferases enzymes (DNMT1, DNMT3).}

Carpel tunnel syndrome (CT) versus Trigger Finger (TF)

In patients with $\mathrm{TF}$, the mRNA expression of DNMT1 was significant ( $p$-value $=0.04$ ) up-regulated $(>50$ folds). While DNMT1 was expressed at a low level in patients with CT syndrome. DNMT3 expression was significant ( $\mathrm{p}$-value= 0.0039) down-regulated in TF when compared to CT patients [Figure.2].

\section{Diabetic TF versus Non-Diabetic TF.}

Diabetes was associated with a statistically significant $(p$-value $=0.04)$ increase in the DNMT1 expression as compared to non-diabetic TF patients. Also, Diabetic TF patients revealed a trend of increase in DNMT3 expression [Figure.4].

\section{Changes in growth factors (TGF-b, IGF) as a result of DNA methylation.}

\section{Carpel tunnel syndrome (CT) versus Trigger Finger (TF).}

The mRNA expressions of Insulin-like growth factors-1 IGF-1 $(p$-value $=0.01))$ and Transforming growth factors $(\mathrm{TGF}-\mathrm{b})(p$-value $=0.011)$ were significantly up-regulated (>250 folds and 70 folds, respectively) in trigger finger, as compared to CT syndrome patients [Figure.1].

\section{Diabetic TF versus Non-Diabetic TF.}

When investigating the IGF-1 and TGF-b expressions in TF and diabetic TF, Diabetes revealed more abnormal statistically significant up-regulation of IGF-1 ( $p$-value= 0.02) and TGF-b ( $p$-value $=0.05)$ growth factors [Figure.3]. 

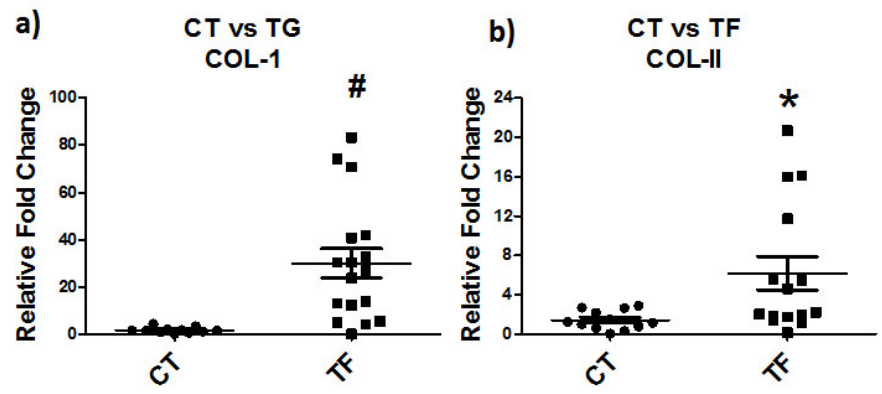

c) $\quad$ CT vs TF
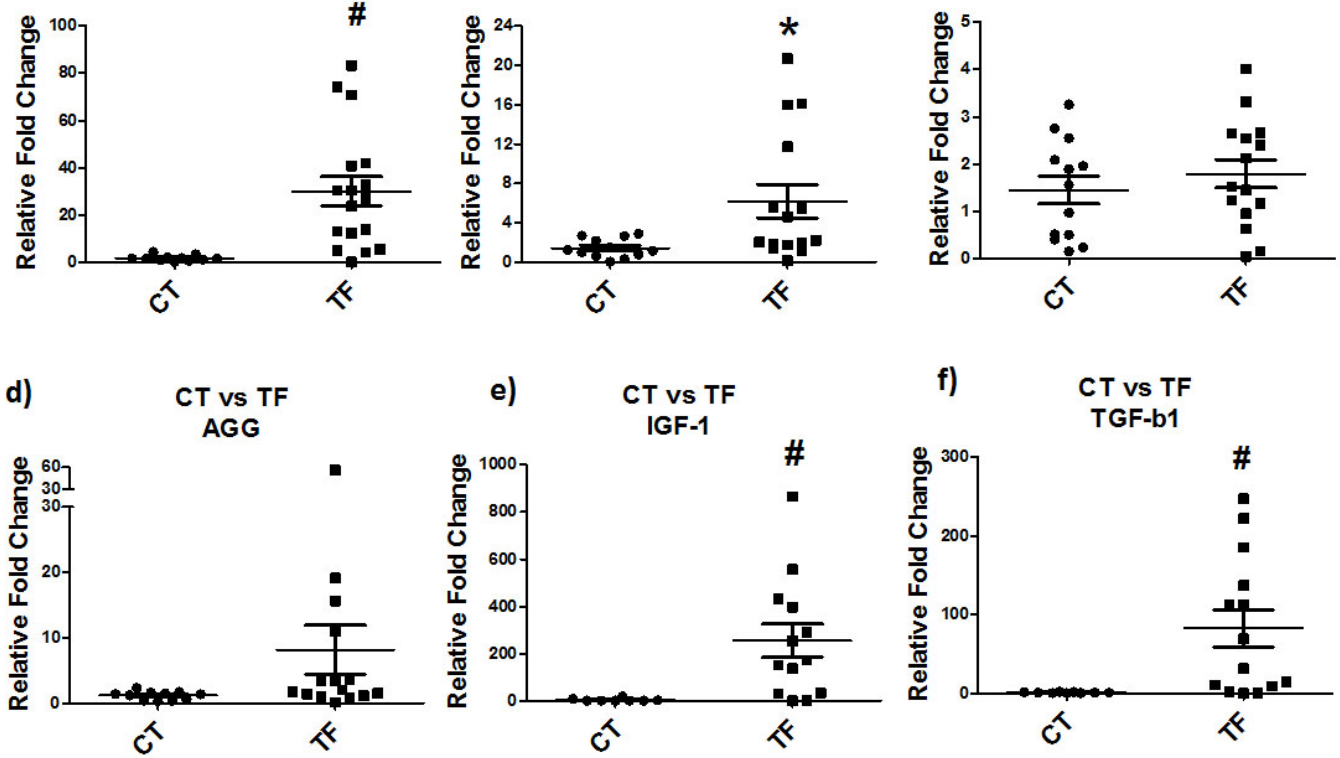

Figure 1: Extracellular matrix and growth factors genes dysregulated in trigger finger samples. (a) Collagen1, (b) Collagen 2, (c) Collagen X, (d) Aggrecan, (e) IGF1 and (f) TGFb1 gene expression level dysregulated in trigger finger (TF) samples compare to Carpel tunnel syndrome (CT). * $P<.05, \# P<.01,(n=8-17)$.

a)
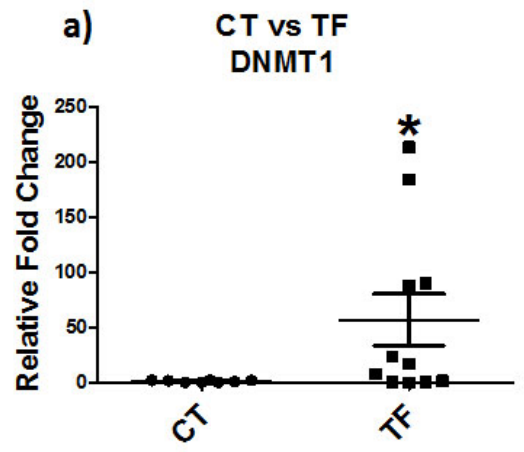

c)

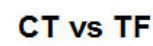

HDAC1

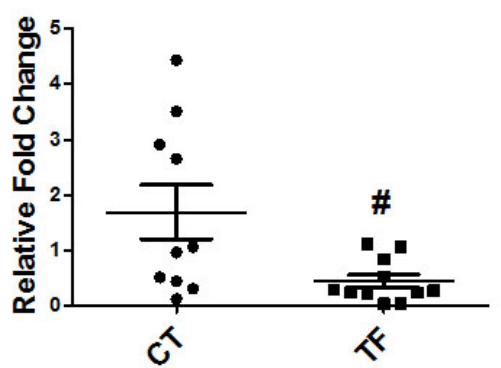

b) CT vs TF

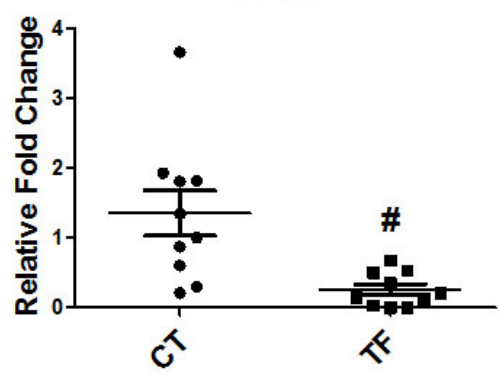

d)

CT vs TF

HDAC2

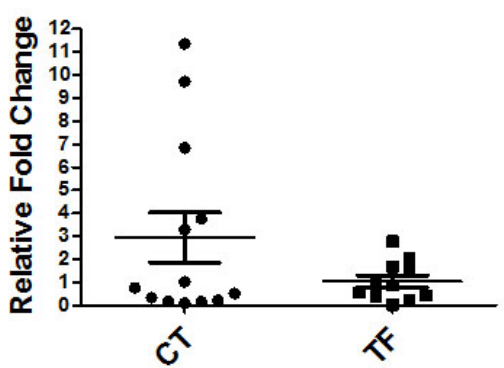

Figure 2: Methylation related genes dysregulated in trigger finger samples. (a) DNMT1, (b) DNMT3, (c) HDAC1, and (d) HDAC2 gene expression level dysregulated in trigger finger (TF) samples compare to Carpel tunnel syndrome (CT). ${ }^{*}<<.05, \# P<.001(n=8-17)$. 

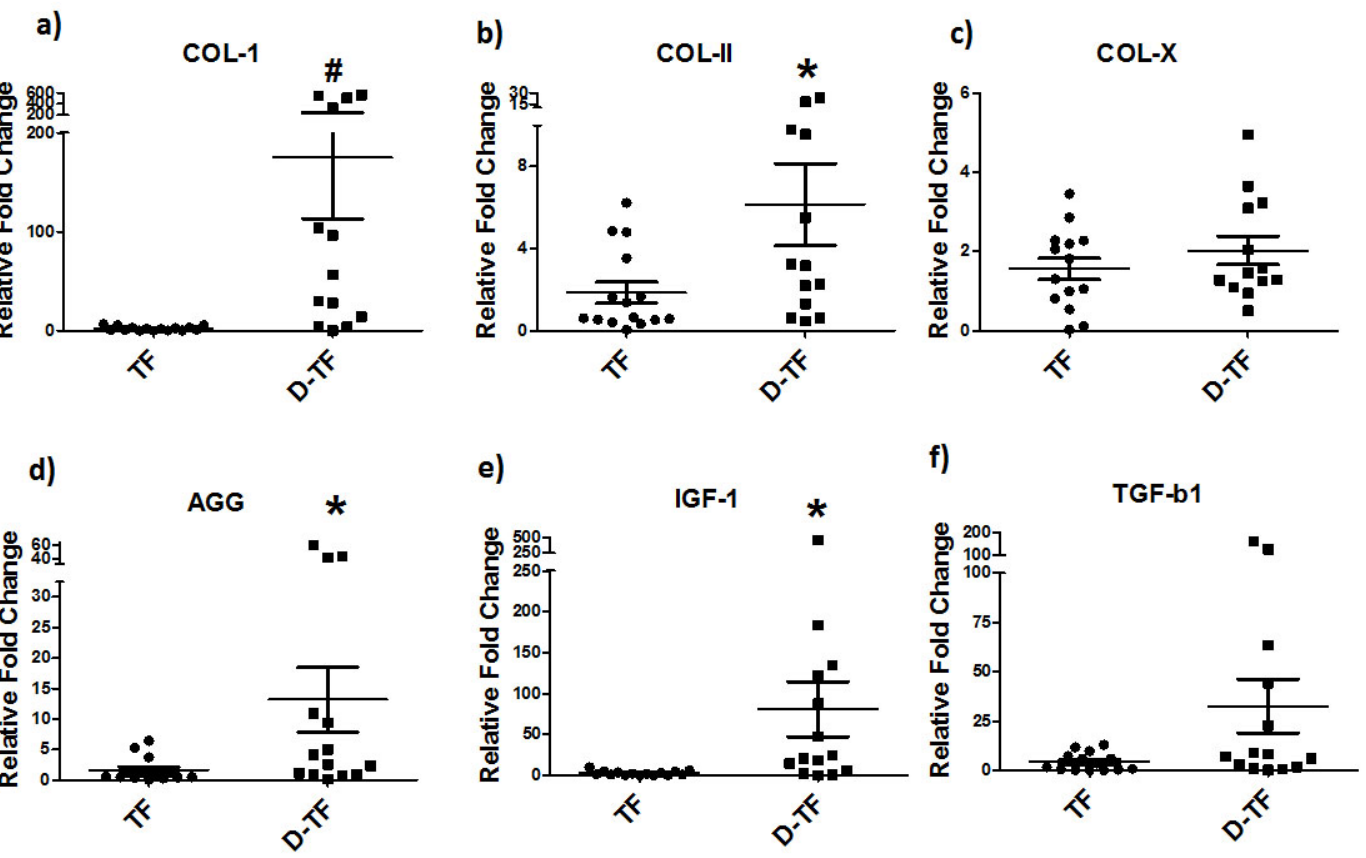

Figure 3: Extracellular matrix and growth factors genes dysregulated in diabetic trigger finger samples. (a) Collagen1, (b) Collagen 2, (c) Collagen X, (d) Aggrecan, (e) IGF1 and (f) TGFb1 gene expression level dysregulated in diabetic trigger finger (D-TF) samples compare to trigger finger $\left({ }^{*} P<.05\right.$, , $\#<<.001(n=8-17)(n=8-17)$.
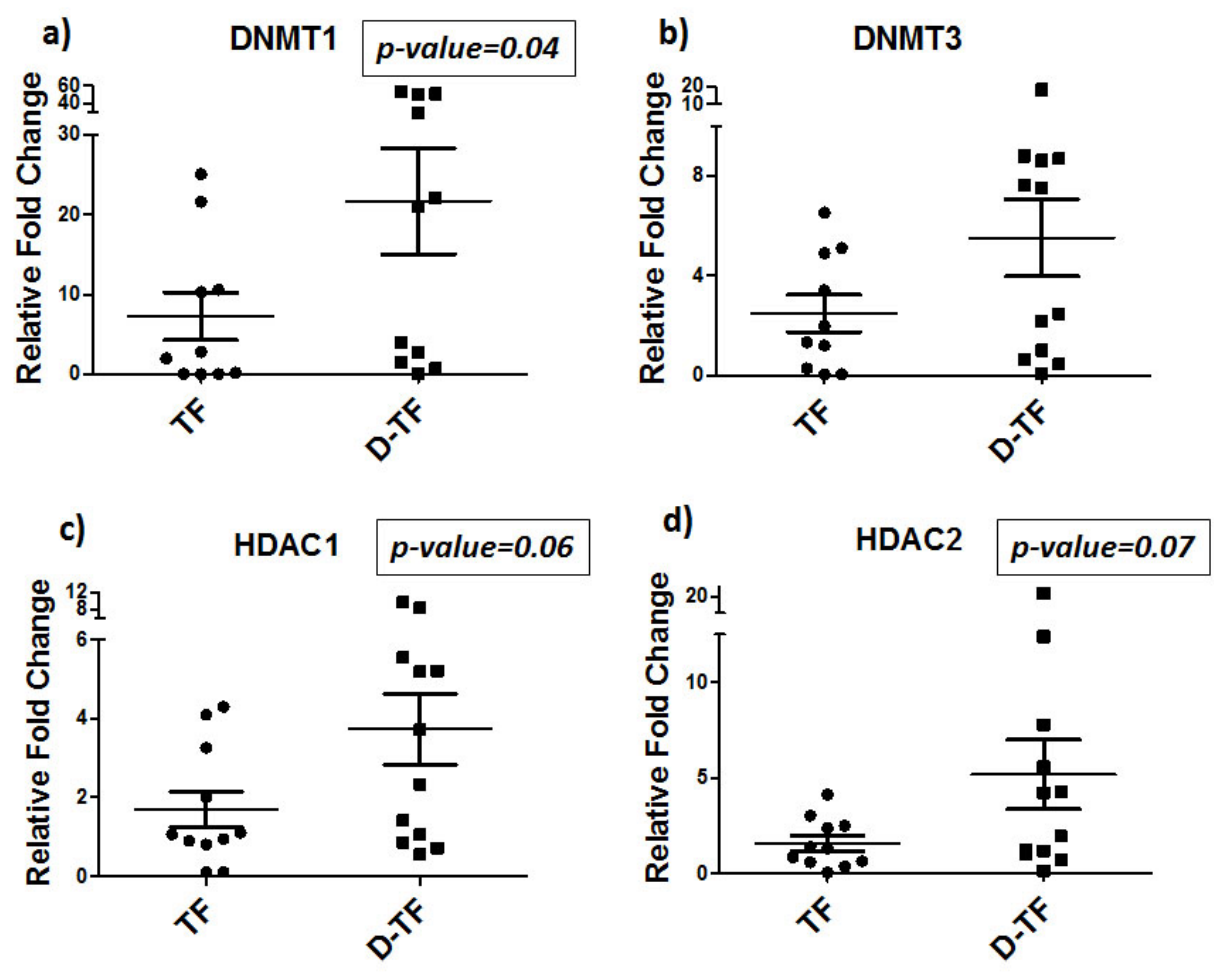

Figure.4: Methylation related genes dysregulated in diabetic trigger finger samples. (a) DNMT1, (b) DNMT3, (c) HDAC1, and (d) HDAC2 gene expression level dysregulated in diabetic trigger finger (D-TF) samples compare to trigger finger (TF) * $\mathrm{P}<.05$, , \#P<.001 ( $\mathrm{n}=8$-17). 
Histone deacetylase enzymes (HDAC1, HDAC2) Carpel tunnel syndrome (CT) versus Trigger Finger (TF).

The histone deacetylase enzymes HDAC1 ( $p$-value= 0.01) was significantly down-regulated, whereas HDAC2 $(p$-value $=0.01)$ showed the trend of down-regulated in TF tissues when compared to CT syndrome [Figure.2].

\section{Diabetic TF versus Non-Diabetic TF.}

HDAC1 $(p$-value $=0.06)$ and HDAC2 $(p$-value $=0.07)$ genes were shown trend of up-regulated in diabetic TF higher than non-diabetic TF [Figure.4].

\section{Discussion}

In this study, we have evaluated the expression of ECM and epigenetic related genes in diabetic and nondiabetic tenosynovitis samples. The overall pattern of gene expression detected in the trigger finger has some similarities to the previous studies on Achilles tendinosis [22-24]. To our knowledge, there is no existing empirical research investigating the various aspects of epigenetic regulation of trigger finger in diabetic and non-diabetic patients. This study presents the first preliminary data of the epigenetic modification of gene expression in diabetic trigger fingers.

The primary determinant of tendinopathy progression is the integrity and remodeling of the extracellular matrix (ECM) [25]. ECM is formed of several structural proteins such as (collagens) and proteoglycans such as (aggrecan, versican, decorin, etc.) [26]. Increase expression of proteoglycans contributes to the alteration of physical properties of the fibrocartilaginous region, which consequently results in changes in pain intensity and threshold in response to mild shear, compression or mechanical load [27-29]. Type I collagen is the most abundant of total body collagen, and it is found in fibrous connective tissues such as tendons [26]. Our results showed significant up-regulation of the mRNA level of COL-I and II within A1 pully of diabetic patients ( $\mathrm{p}=0.0164$ and $p=0.0351$, respectively). Also, Aggrecan expression is substantially and abnormally up-regulated in diabetic TF. Chronic hyperglycemia in diabetes increased the production of collagen and other extracellular matrix components, which in turn are deposited in a disorganized manner [30]. One of the hypothesized effects of corticosteroid injection in releasing the trigger finger is minimally restoring collagen hemostasis via increasing expression of organized collagen without any structural disruption to the tendon integrity [31]. The disruption of ECM and its compactness in diabetic tendinopathy may explain the higher risk of resistance to corticosteroid injections and the recurrence of TF in diabetes.

There are many factors that regulate the cellular and extracellular mechanisms of proteoglycan synthesis. The changes in homeostasis (formation/degradation balance) of ECM molecules could ultimately alter the composition and physical properties [32]. All these cellular and extracellular components are targeted by circulating factors such as growth factors, cytokines, and hormones. The Transforming growth factor- $\beta$ (TGF- $\beta$ ) and Insulinlike growth factor-I (IGF-I) can each act as local mediators of cellular response, and they can regulate cellular interactions based on the ubiquitous nature of their receptors [33]. TGF- $\beta$ affects all phases of the healing process [34] TGF- $\beta$ is mitogenic for fibroblasts and stimulates the production of collagen and fibronectin. Its effects on the extracellular matrix are more complex than that of any other growth factor. TGF-b and IGF-1 have been specifically involved in the regulation of cartilage development [35]. Also, in-vivo studies have correlated the increased TGF-b expression and increased synthesis of ECM [36,37]. Our results revealed that the mRNA expressions of Insulin-like growth factors-1 (IGF-1) and Transforming growth factors (TGF-b) were abnormally up-regulated in both diabetic and non-diabetic TF, with higher levels in diabetes. Local overexpression of TGF-b in the synovium leads to the formation of cartilage-like tissue between the collateral ligaments and bone, synovial hyperplasia, as well as the formation of chondo-osteophytes [38].

Epigenetic regulation has been specifically implicated in the regulation and pathogenesis of many musculoskeletal disorders. Epigenetics is an acquired modification of chromatin DNA or histone proteins such as DNA methylation and histone modification. These modifications mainly dysregulated gene expression without an alteration in the DNA sequence [39]. DNA methylation plays an important role in the regulation of inflammatory genes. Also, its role in the regulation of type-I and II collagen genes during chondrocyte differentiation and dedifferentiation are well known [40]. DNA methylation is mediated by two main enzymes, DNA methyltransferases 1 and 3 (DNMT1, DNMT3), while Histones modulate the activity of gene promotor by histone acetyltransferase (HAT) and histone deacetylases (HDAC), respectively [41]. Aberrant DNA methylation and histone modification can be induced during aging and chronic inflammation. Our results showed that the mRNA 
expression of DNMT1 was highly up-regulated ( $>50$ folds) in patients with TF. While Diabetic TF is associated with a statistically significant increase in the DNMT3 expression compared to non-diabetic TF patients. $(\mathrm{p}=0.0485)$. Besides, Diabetic TF patients revealed a slight increase in DNMT1 expression. ( $\mathrm{p}=0.1144)$. Yan et al [42] demonstrated that diabetes impairs the healing by DNMT1-dependent dysregulation of hematopoietic stem cell differentiation towards macrophages. Several other studies also reported dysregulation of DNMTs in diabetic complications such as diabetic retinopathy [43], neuropathy [44], nephropathy [45], and podocyte injury [45].

Histone deacetylase enzymes are known to plays a critical role in embryonic development, tissue homeostasis, and pathophysiology of various diseases [46]. We analyze two main histone deacetylase enzymes, HDAC1 and HDAC2. Both enzymes were significantly up-regulated in diabetic TF, higher compared to nondiabetic TF. Noh et al. (2009) reported HDAC-2 activity elevated in the kidneys of STZ-induced diabetic rats and $\mathrm{db} / \mathrm{db}$ mice, whereas treatment with nonselective HDAC inhibitor decreased accumulation of extracellular matrix and prevented epithelial-to-mesenchymal transition and decrease fibrosis in the diabetic kidney [47]. Similarly, Lee et al. (2019) demonstrated that HDAC inhibitor (MGCD0103), ameliorated streptozotocin (STZ)-induced hyperglycemia, islet deformation, decreased insulin levels, macrophage infiltration and protects pancreas from STZ-induced oxidative stress [48]. Several DNA methyltransferases and histone deacetylase inhibitors are approved by the FDA to treat various cancers [49,50]. Recent preclinical studies showed promising positive outcomes in diabetic complications $[47,48,51,52]$. Of note, this may highlight the clinical relevance of DNMT and HDAC inhibitors as a novel approach to manage diabetic TF. Combination of DNA methyltransferases and histone deacetylase inhibitors, along with anti-diabetic drugs, might help in reducing the diabetic complication of TF.

Our study has some limitations. First, we couldn't provide clinical data about the trigger finger stages. Secondly, the control specimens were obtained from patients with carpal tunnel syndrome, among which the incidence of trigger finger is known to be high, and third, we only analyze gene expression at the mRNA level. It is impossible to collect specimens from healthy subjects. Furthermore, the sample collected from patients is a small quantity, which is not sufficient for both gene and protein analysis.

Diabetes is sufficient to induce an irreversible cascade of pathologic changes in the tendon structure, including altered ECM organization, ultimately leading to diminished mechanical properties and tendon range of motion. The chronic state of hyperglycemia induces epigenetic modifications of gene expressions. This seems to significantly impact the development, recurrence, and progression of trigger finger in diabetic patients. Understanding the alterations in histone modifications and DNA methylation will provide a good base for better managing trigger finger cases and even developing novel, targeted therapeutic drugs. Further studies with large patient samples and wide epigenetic analysis are still required to define the role of epigenetics modifications in trigger fingers. Taken together, these insights into the mechanism by which diabetes impairs the trigger finger management open multiple avenues to new promising techniques to restore normal function and thereby reduce the risk of resistance to conservative therapy or recurrence in diabetic patients.

Conflict of interests: The authors declare no conflict of interests.

Authors Contributions: Designed and coordinated the study; Sadanand Fulzele, Mark Fulcher, Carlos Isales. Performed the experiments, acquired and analyzed data; Michel Cain, Ashis K. Mondal, Ravindra Kolhe, Umar Ghilzai; Wrote and edited the manuscript; Sadanand Fulzele, Mohamed E. Awad, Carlos Isales.

Acknowledgments: We would like to thank The Department of Orthopaedic Surgery for their support.

\section{References}

1. Moore JS. Flexor tendon entrapment of the digits (trigger finger and trigger thumb). J Occup Environ Med. 2000 May;42(5):52645.

2. Ryzewicz M, Wolf JM. Trigger digits: principles, management, and complications. J Hand Surg Am. 2006 Jan;31(1):135-46.

3. Lundin AC, Eliasson P, Aspenberg P. Trigger finger and tendinosis. J Hand Surg Eur Vol. 2012 Mar;37(3):233-6.

4. McAuliffe JA. Tendon disorders of the hand and wrist. J Hand Surg Am. 2010 May;35(5):846-53.

5. Bonnici AV, Spencer JD. A survey of 'trigger finger' in adults. J Hand Surg [Br]. 1988 May;13(2):202-3.

6. Weilby A. Trigger finger. Incidence in children and adults and the possibility of a predisposition in certain age groups. Acta Orthop Scand. 1970;41(4):419-27.

7. Peters-Veluthamaningal C, van der Windt DA, Winters JC, Meyboom-de Jong B. Corticosteroid injection for trigger finger in adults. Cochrane Database Syst Rev. 2009 Jan;(1):CD005617.

8. Wessel LE, Fufa DT, Boyer MI, Calfee RP. Epidemiology of carpal tunnel syndrome in patients with single versus multiple trigger digits. J Hand Surg Am. 2013 Jan;38(1):49-55. 
9. Stahl S, Kanter Y, Karnielli E. Outcome of trigger finger treatment in diabetes. J Diabetes Complications. 1997 Sep-Oct;11(5):28790.

10. Grandizio LC, Beck JD, Rutter MR, Graham J, Klena JC. The incidence of trigger digit after carpal tunnel release in diabetic and nondiabetic patients. J Hand Surg Am. 2014 Feb;39(2):2805.

11. Douloumpakas I, Pyrpasopoulou A, Triantafyllou A, Sampanis C, Aslanidis S. Prevalence of musculoskeletal disorders in patients with type 2 diabetes mellitus: a pilot study. Hippokratia. 2007 Oct;11(4):216-8.

12. Abate M, Schiavone C, Salini V, Andia I. Occurrence of tendon pathologies in metabolic disorders. Rheumatology (Oxford). 2013 Apr;52(4):599-608.

13. Anderson B, Kaye S. Treatment of flexor tenosynovitis of the hand ('trigger finger') with corticosteroids. A prospective study of the response to local injection. Arch Intern Med. 1991 Jan;151(1):153-6.

14. Benson LS, Ptaszek AJ. Injection versus surgery in the treatment of trigger finger. J Hand Surg Am. 1997 Jan;22(1):138-44.

15. Murphy D, Failla JM, Koniuch MP. Steroid versus placebo injection for trigger finger. J Hand Surg Am. 1995 Jul;20(4):62831.

16. Zyluk A, Jagielski G. Percutaneous A1 pulley release vs steroid injection for trigger digit: the results of a prospective, randomized trial. J Hand Surg Eur Vol. 2011 Jan;36(1):53-6.

17. Mol MF, Neuhaus V, Becker SJ, Jupiter JB, Mudgal C, Ring D. Resolution and recurrence rates of idiopathic trigger finger after corticosteroid injection. Hand (N Y). 2013 Jun;8(2):183-90.

18. Grandizio LC, Speeckaert A, Brothers J, Graham J, Klena JC. Predictors of Recurrence After Corticosteroid Injection for Trigger Digits. Hand (N Y). 2017 Jul;12(4):352-6.

19. Rorbach-Dolata A, Kubis A, Piwowar A. Epigenetic modifications: an important mechanism in diabetic disturbances. Postepy Hig Med Dosw. 2017 Nov;71(0):960-74.

20. Bayarsaihan D. Epigenetic mechanisms in inflammation. J Dent Res. 2011 Jan;90(1):9-17.

21. Lundin AC, Aspenberg P, Eliasson P. Trigger finger, tendinosis, and intratendinous gene expression. Scand J Med Sci Sports. 2014 Apr;24(2):363-8.

22. Corps AN, Robinson AH, Movin T, Costa ML, Hazleman BL, Riley GP. Increased expression of aggrecan and biglycan mRNA in Achilles tendinopathy. Rheumatology (Oxford). 2006 Mar;45(3):291-4

23. de Mos M, van El B, DeGroot J, Jahr H, van Schie HT, van Arkel ER, et al. Achilles tendinosis: changes in biochemical composition and collagen turnover rate. Am J Sports Med. 2007 Sep;35(9):1549-56.

24. Ireland D, Harrall R, Curry V, Holloway G, Hackney R, Hazleman $B$, et al. Multiple changes in gene expression in chronic human Achilles tendinopathy. Matrix Biol. 2001 Jun;20(3):159-69.

25. Eriksen HA, Pajala A, Leppilahti J, Risteli J. Increased content of type III collagen at the rupture site of human Achilles tendon. J Orthop Res. 2002 Nov;20(6):1352-7.

26. Kannus P. Structure of the tendon connective tissue. Scand J Med Sci Sports. 2000 Dec;10(6):312-20.

27. Benjamin, M. and J.R. Ralphs, Fibrocartilage in tendons and ligaments - an adaptation to compressive load. 1998. 193(Pt 4): p. 481-94.
28. Robbins JR, Evanko SP, Vogel KG. Mechanical loading and TGFbeta regulate proteoglycan synthesis in tendon. Arch Biochem Biophys. 1997 Jun;342(2):203-11.

29. Thomopoulos S, Williams GR, Gimbel JA, Favata M, Soslowsky LJ. Variation of biomechanical, structural, and compositional properties along the tendon to bone insertion site. J Orthop Res. 2003 May;21(3):413-9.

30. Studentsova V, et al. Obesity/Type II Diabetes Promotes Function-Limiting Changes In Flexor Tendon Extracellular Matrix Organization That Are Not Reversed By Restoring Normal Metabolic Function. bioRxiv, 2017: p. 143149.

31. Wei AS, Callaci JJ, Juknelis D, Marra G, Tonino P, Freedman KB, et al. The effect of corticosteroid on collagen expression in injured rotator cuff tendon. J Bone Joint Surg Am. 2006 Jun;88(6):13318.

32. Nathan C, Sporn M. Cytokines in context. J Cell Biol. 1991 Jun;113(5):981-6.

33. Turley JM, Falk LA, Ruscetti FW, Kasper JJ, Francomano T, Fu T, et al. Transforming growth factor beta 1 functions in monocytic differentiation of hematopoietic cells through autocrine and paracrine mechanisms. Cell Growth Differ. 1996 Nov;7(11):153544.

34. Yang L, Qiu CX, Ludlow A, Ferguson MW, Brunner G. Active transforming growth factor-beta in wound repair: determination using a new assay. Am J Pathol. 1999 Jan;154(1):105-11.

35. S., J., et al., Transforming growth factor- $\beta 1$ and fibroblast growth factors in rat growth plate. Journal of Orthopaedic Research, 1995. 13(5): p. 761-768.

36. Border WA, Noble NA, Yamamoto T, Harper JR, Yamaguchi Y, Pierschbacher MD, et al. Natural inhibitor of transforming growth factor- $\beta$ protects against scarring in experimental kidney disease. Nature. 1992 Nov;360(6402):361-4.

37. Westergren-Thorsson G, Hernnäs J, Särnstrand B, Oldberg A, Heinegård D, Malmström A. Altered expression of small proteoglycans, collagen, and transforming growth factor-beta 1 in developing bleomycin-induced pulmonary fibrosis in rats. J Clin Invest. 1993 Aug;92(2):632-7.

38. Bakker AC, van de Loo FA, van Beuningen HM, Sime P, van Lent $\mathrm{PL}$, van der Kraan PM, et al. Overexpression of active TGF-beta-1 in the murine knee joint: evidence for synovial-layer-dependent chondro-osteophyte formation. Osteoarthritis Cartilage. 2001 Feb;9(2):128-36.

39. Kelly TK, De Carvalho DD, Jones PA. Epigenetic modifications as therapeutic targets. Nat Biotechnol. 2010 Oct;28(10):1069-78.

40. Fernández MP, Young MF, Sobel ME. Methylation of type II and type I collagen genes in differentiated and dedifferentiated chondrocytes. J Biol Chem. 1985 Feb;260(4):2374-8.

41. Gabay O, Clouse KA. Epigenetics of cartilage diseases. Joint Bone Spine. 2016 Oct;83(5):491-4.

42. Yan, J., et al., diabetes impairs wound healing by Dnmt1dependent dysregulation of hematopoietic stem cells differentiation towards macrophages. Nature Communications, 2018. 9(1): p. 33. 43.

43. Mishra M, Kowluru RA. The role of DNA methylation in the metabolic memory phenomenon associated with the continued progression of diabetic retinopathy. Invest Ophthalmol Vis Sci. 2016 Oct;57(13):5748-57.

44. Guo K, Eid SA, Elzinga SE, Pacut C, Feldman EL, Hur J. Genomewide profiling of DNA methylation and gene expression 
identifies candidate genes for human diabetic neuropathy. Clin Epigenetics. 2020 Aug;12(1):123.

45. Zhang L, Zhang Q, Liu S, Chen Y, Li R, Lin T, et al. DNA methyltransferase 1 may be a therapy target for attenuating diabetic nephropathy and podocyte injury. Kidney Int. 2017 Jul;92(1):140-53.

46. Delcuve GP, Khan DH, Davie JR. Roles of histone deacetylases in epigenetic regulation: emerging paradigms from studies with inhibitors. Clin Epigenetics. 2012 Mar;4(1):5.

47. Noh H, Oh EY, Seo JY, Yu MR, Kim YO, Ha H, et al. Histone deacetylase-2 is a key regulator of diabetes- and transforming growth factor-beta1-induced renal injury. Am J Physiol Renal Physiol. 2009 Sep;297(3):F729-39.

48. Lee HA, Lee E, Do GY, Moon EK, Quan FS, Kim I. Histone deacetylase inhibitor MGCD0103 protects the pancreas from streptozotocin-induced oxidative stress and $\beta$-cell death. Biomed Pharmacother. 2019 Jan;109:921-9.

49. Ganesan A, Arimondo PB, Rots MG, Jeronimo C, Berdasco M. The timeline of epigenetic drug discovery: from reality to dreams. Clin Epigenetics. 2019 Dec;11(1):174.

50. Martinez-Moreno JM, Fontecha-Barriuso M, Martin-Sanchez D, Guerrero-Mauvecin J, Goma-Garces E, Fernandez-Fernandez B, et al. Epigenetic Modifiers as Potential Therapeutic Targets in Diabetic Kidney Disease. Int J Mol Sci. 2020 Jun;21(11):4113.

51. Martinez-Moreno JM, Fontecha-Barriuso M, Martin-Sanchez D, Guerrero-Mauvecin J, Goma-Garces E, Fernandez-Fernandez B, et al. Epigenetic Modifiers as Potential Therapeutic Targets in Diabetic Kidney Disease. Int J Mol Sci. 2020 Jun;21(11):4113.

52. Arguelles AO, Meruvu S, Bowman JD, Choudhury M. Are epigenetic drugs for diabetes and obesity at our door step? Drug Discov Today. 2016 Mar;21(3):499-509. 